03,05

\title{
Влияние размерного квантования на спиновую поляризацию дырок в структурах с квантовыми ямами разбавленного магнитного полупроводника (Ga,Mn)As/AlAs
}

\author{
(C) Г.С. Димитриев ${ }^{1}$, В.Ф. Сапега ${ }^{1}$, Н.С. Аверкиев ${ }^{1}$, И.Е. Панайотти ${ }^{1}$, К.Н. Ploog ${ }^{2}$ \\ ${ }^{1}$ Физико-технический институт им. А.Ф. Иофрер РАН, \\ Санкт-Петербург, Россия \\ ${ }^{2}$ Paul-Drude-Institut für Festkörperelektronik, \\ Berlin, Germany \\ E-mail: dimitriev@mail.ioffe.ru
}

(Поступила в Редакцию 3 апреля 2017 г.)

Исследовано влияние размерного квантования на спиновую поляризацию дырок в структуре с квантовыми ямами ферромагнитного полупроводника ( $\mathrm{Ga}, \mathrm{Mn}) \mathrm{As}$. Показано, что спиновая поляризация дырок в примесной зоне определяется, скорее, магнитными свойствами самого GaMnAs, а не эффектом размерного квантования. Развита модель акцептора Mn в квантовой яме, описывающая поляризационные характеристики фотолюминесценции в квантовых ямах GaAs : Mn/AlAs. Экспериментальные данные и теоретический анализ продемонстрировали, что спиновая поляризация дырок в квантовых ямах (Ga,Mn)As/AlAs может быть объяснена в модели, предполагающей, что дырки локализованы в примесной зоне.

Работа поддержана грантом РФФИ № 15-52-12017 ННИО-а и грантом Правительства РФ (договор № 14.Z50.31.0021, 07.04.2014-31.12.2018. Ведущий ученый М.Х. Байер).

DOI: 10.21883/FTT.2017.11.45068.109

\section{1. Введение}

Размерное квантование носителей заряда в квантовых ямах играет важную роль в физике полупроводников, поскольку существенно изменяет энергетический спектр носителей и приводит к перенормировке фундаментальных характеристик, таких как эффективная масса и $g$-фактор, а также увеличивает обменное взаимодействие. Можно ожидать, что размерное квантование повлияет на обменное взаимодействие и магнитные свойства разбавленного магнитного полупроводника (РМП) $(\mathrm{Ga}, \mathrm{Mn}) \mathrm{As}$, который привлекает растущий интерес c момента его открытия в 1996 [1]. Влияние размерного квантования на знак и величину $s$ - $d$ обмена в структурах с квантовыми ямами (СКЯ) GaAs:Mn/AlAs было исследовано в работах [2,3]. Влияние размерного квантования на намагниченность и магнитооптические свойства было исследовано в СКЯ РМП (Ga,Mn)As/AlAs [4]. В этой работе было показано, что квантование приводит к возникновению намагниченности, лежащей вне плоскости СКЯ. Этот эффект дает возможность управлять магнитными свойствами и спиновой поляризацией носителей заряда в СКЯ. С другой стороны, изучение одновременного влияния размерного квантования и ферромагнитного упорядочения на спиновую поляризацию позволяет непосредственно проверить модель ферромагнетизма (Ga,Mn)As/AlAs PMП.

В настоящей работе приведены результаты исследования спиновой поляризации дырок в ферромагнитных квантовых ямах (КЯ) методом поляризованной фотолюминесценции горячих электронов (ГФЛ) [5,6]. Показано, что переход от объемного РМП к двумерному сопро- вождается существенным изменением поляризации ГФЛ в магнитном поле, причем в отсутствие внутренних локальных полей деформации или электрических, можно было бы ожидать заметного увеличения спиновой поляризации дырок на акцепторе или примесной зоны вплоть до 70\%. Однако оказалось, что спиновая поляризация дырок в двумерном РМП в большей степени определяется случайными полями, а не размерным квантованием. Показано, что ферромагнетизм в КЯ РМП (Ga,Mn)As/AlAs обусловлен скорее дырками акцепторной примесной зоны, чем свободными дырками валентной зоны.

\section{2. Образцы и экспериментальная установка}

Структуры с квантовыми ямами (Ga,Mn)As/AlAs были выращены на полуизолирующей подложке $\mathrm{GaAs}(001)$ с помощью стандартной молекулярно-пучковой эпитаксии (МПЭ) [7]. Толщина буферного слоя составляла $330 \mathrm{~nm}$. Затем СКЯ выращивались при температуре подложки $250^{\circ} \mathrm{C}$ и со скоростью роста $24 \mathrm{~nm} / \mathrm{h}$. Все выращенные гетероструктуры состояли из 30 периодов $\mathrm{Ga}_{1-x} \mathrm{Mn}_{x} \mathrm{As} / \mathrm{AlAs}$ c $x=0.025$ и 0.04 и толщиной ямы, изменявшейся в интервале $5-12.5 \mathrm{~nm}$ в случае слоев $\mathrm{Ga}_{1-x} \mathrm{Mn}_{x} \mathrm{As}$ и в интервале 3-6 nm для барьеров AlAs. Аналогично СКЯ с содержанием Mn на уровне легирования (около $5 \cdot 10^{17} \mathrm{~cm}^{-3}$ ) были выращены при $T_{s}=560^{\circ} \mathrm{C}$. Для сравнения был выращен (при $T_{s}=560^{\circ} \mathrm{C}$ ) объемный образец $\mathrm{GaAs}: \mathrm{Mn}$ толщиной $1000 \mathrm{~nm}$ и с такой же концентрацией Mn. Наконец, 
Параметры структур с квантовыми ямами GaAs:Mn и $\mathrm{Mn}_{x} \mathrm{Ga}_{1-x}$ As РМП, использованные в настоящей работе (содержание Mn, ширина ямы/ширина барьера, а также тип магнетизма парамагнитный (ПМ) или ферромагнитный $(\Phi \mathrm{M})$ СКЯ и температура Кюри $T_{C}$, измеренная методом SQUID)

\begin{tabular}{c|c|c|c|c}
\hline Образец & $\begin{array}{c}\text { Концентрация } \\
\text { Mn }\end{array}$ & $\begin{array}{c}\text { Яма } \\
\text { (слой)/ширина } \\
\text { барьера (nm) }\end{array}$ & $\begin{array}{c}\text { Магнитная } \\
\text { фаза }\end{array}$ & $T_{C}(K)$ \\
\hline$R 1$ & $1.5 \cdot 10^{-5}$ & 1000 & ПМ & - \\
$D 1$ & $2.5 \cdot 10^{-5}$ & $(10 / 6)$ & ПМ & - \\
$D 2$ & $2.5 \cdot 10^{-5}$ & $(5 / 3)$ & ПM & - \\
FMR & 0.04 & 1000 & $\Phi M$ & 55 \\
FM1 & 0.025 & $(10 / 3)$ & $\Phi M$ & 35 \\
FM2 & 0.04 & $(10 / 3)$ & $\Phi M$ & 30 \\
FM3 & 0.025 & $(5 / 3)$ & $\Phi M$ & 6
\end{tabular}

образцы объемного разбавленного магнитного полупроводника $(\mathrm{Ga}, \mathrm{Mn}) \mathrm{As}$ толщиной $1000 \mathrm{~nm}$ выращивались при $T_{s}=250^{\circ} \mathrm{C}$. Содержание $\mathrm{Mn}$ в РМП $(\mathrm{Ga}, \mathrm{Mn}) \mathrm{As}$ составляло $2.5 \%$ и $4 \%$. Параметры исследованных образцов приведены в таблице.

Для возбуждения люминесценции использовались линии $\mathrm{He}-\mathrm{Ne}(632.8 \mathrm{~nm}), \mathrm{Kr}(676.4 \mathrm{~nm})$ и $\operatorname{Ar}(488 \mathrm{~nm})$ лазеров. Плотность энергии луча, сфокусированного на образце, составляла от 5 до $50 \mathrm{~W} \mathrm{~cm}^{-2}$. Спектры ГФЛ измерялись на спектрографе DILOR (c CCD камерой) или монохроматоре Jobin-Yvon U-1000 (с фотоумножителем) при температуре $5 \mathrm{~K}$ и в магнитном поле до $10 \mathrm{~T}$ в геометрии на отражение Фарадея и Фойхта, используя установку, описанную в работе [6].

Степень циркулярной поляризации ГФЛ в геометрии Фарадея определялась с помощью стандартного выражения $\rho_{c}=\left(I^{+}-I^{-}\right) /\left(I^{+}+I^{-}\right)$, где $I^{+}$и $I^{-}-$ интенсивности люминесценции, измеренные в $\sigma^{+}$и $\sigma^{-}$ поляризациях соответственно. В геометрии Фойхта измерялась линейная поляризация люминесценции при неполяризованном возбуждении. В этом случае измерялись интенсивности люминесценции $I_{\|}$и $I_{\perp}$, измеренные в осях параллельных и перпендикулярных магнитному полю $\mathbf{B}$ соответственно. Для определения степени поляризации использовалось выражение $\rho_{l}=\left(I_{\|}-I_{\perp}\right)\left(I_{\|}+I_{\perp}\right)$. При этом $\rho_{l}$ измерялась для двух ориентаций магнитного поля, т. е. или $B \|[100]$, или $B \perp[100]$.

\section{3. Экспериментальные результаты}

На рис. 1 показан спектр ГФЛ, полученный на легированном образце GaAs : Mn/AlAs $\left(N_{\mathrm{Mn}} \sim 5 \cdot 10^{-17} \mathrm{~cm}^{-3}\right)$ и $(\mathrm{Ga}, \mathrm{Mn}) \mathrm{As} / \mathrm{AlAs}(x=2.5 \%)(10 / 3) \mathrm{nm}$ при возбуждении $\mathrm{He}-\mathrm{Ne}$-лазером $\left(\hbar \omega_{e x}=1.96 \mathrm{eV}\right)$ и при температуре $T=5 \mathrm{~K}$. Спектр легированного образца $D 1$ состоит из полосы ФЛ с энергией $1.441 \mathrm{eV}$, обусловленной рекомбинацией термализованных свободных электронов первой подзоны размерного квантования с дырками, связанными на нейтральном акцепторе $\mathrm{Mn}\left(1 e-A^{0}\right)$. Линии ФЛ, обозначенные как $2 e-A^{0}$ и $3 e-A^{0}$ связаны с рекомбинацией электронов со дна второй и третьей подзон размерного квантования с дырками, связанными на нейтральном акцепторе Mn. Межзонная рекомбинация электронов и дырок $(1 e-1 h h)$ перекрывается с линией ФЛ $2 e-A^{0}$. Линия ФЛ $E_{g}+\Delta$ обусловлена рекомбинацией электронов первой подзоны размерного квантования с дырками спин-отщепленной зоны [6]. ГФЛ всех СКЯ на основе РМП $(\mathrm{Ga}, \mathrm{Mn}) \mathrm{As}$ (в качестве примера см. спектр образца FM2 на рис. 1) представляют из себя бесструктурный спектр, который начинается ниже по энергии, чем линия лазера. Заштрихованные линии на этом спектре соответствуют краевой люминесценции подложки GaAs. Как и в случае объемного $(\mathrm{Ga}, \mathrm{Mn})$ As краевая фотолюминесценция ферромагнитной СКЯ сильно подавлена из-за быстрой безызлучательной рекомбинации [6]. Резкое падение на высокоэнергетичном краю спектра (обозначен стрелкой со значком „о“) ГФЛ каждого из образцов соответствует рекомбинации фотовозбужденных электронов из точки рождения с дыркой, связанной

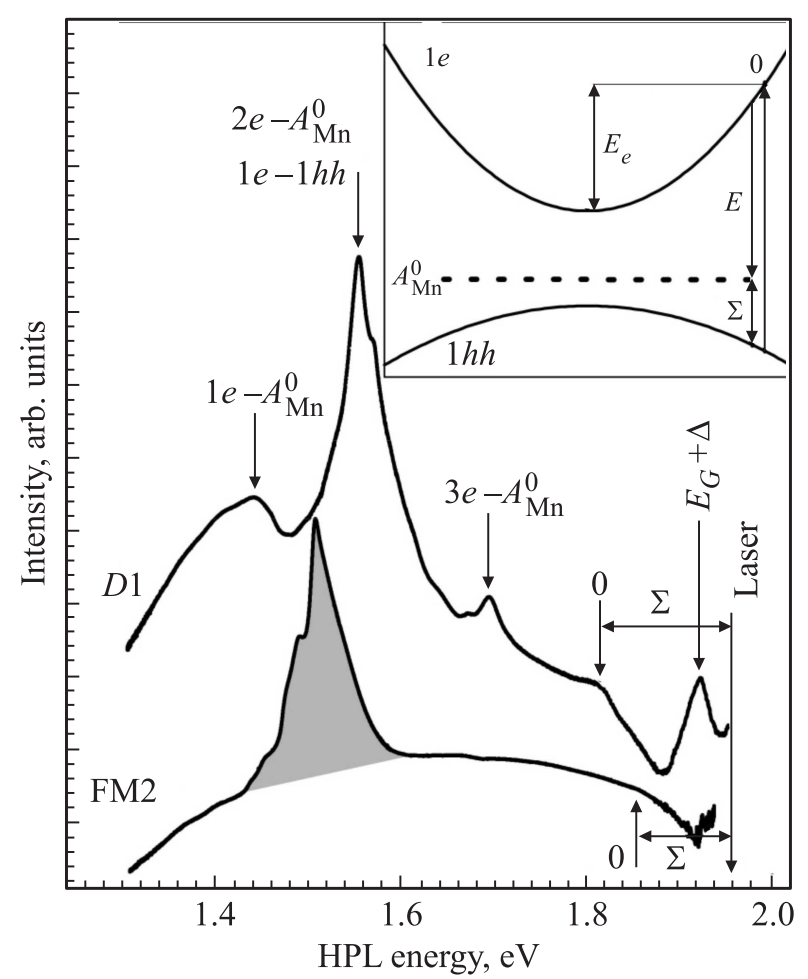

Рис. 1. Спектры ГФЛ СКЯ (Ga,Mn)As образцов $D 1$ и FM2, полученные при возбуждении лазером с энергией $\hbar \omega_{e x}=1.96 \mathrm{eV}$ при $T=5 \mathrm{~K}$. Стрелки, обозначенные ' 0 ' показывают энергию фотонов, испущенных при рекомбинации электронов из точки рождения. Рекомбинация равновесных электронов из первой, второй и третьей зон размерного квантования с дырками, связанными на акцепторе $\mathrm{Mn}$ обозначены стрелками $1 e-A_{\mathrm{Mn}}^{0}$, $2 e-A_{\mathrm{Mn}}^{0}$ и $3 e-A_{\mathrm{Mn}}^{0}$ соответственно. Полоса люминесценции, обусловленная рекомбинацией электронов, связанных на двойном доноре Mn с дырками подзоны, отщепленной спин-орбитальным взаимодействием, обозначена как $E_{G}+\Delta$. Вставка объясняет природу ГФЛ. 


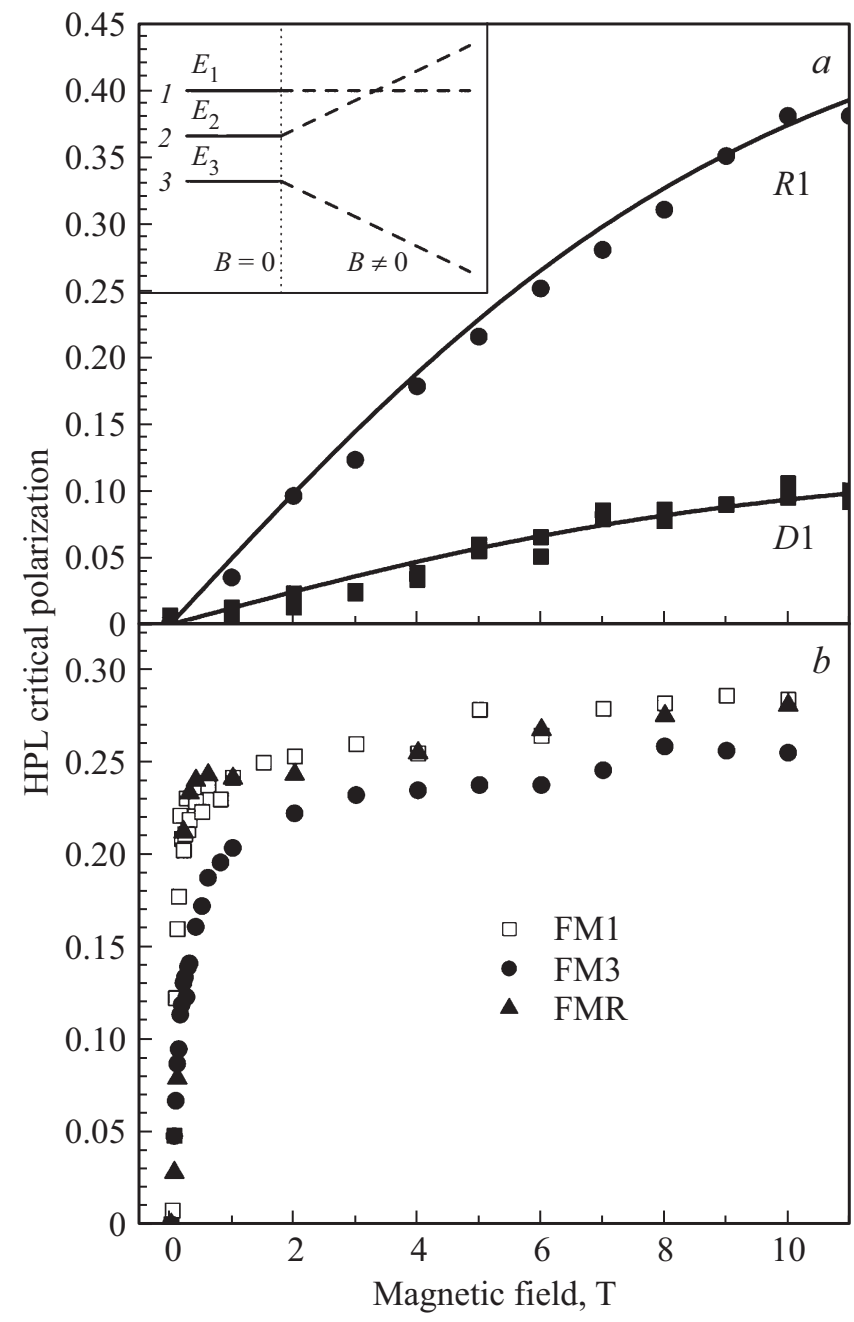

Рис. 2. Зависимость циркулярной поляризации ГФЛ от магнитного поля: $(a)$ измеренная в геометрии Фарадея при температуре $T=5 \mathrm{~K}$ в легированном объемном образце $R 1$ (заштрихованные круги) и СКЯ $D 1$ (заштрихованные квадраты) образцах. Сплошная кривая - аппроксимация с использованием результатов работы [8] для образцов $R 1$ и $D 1$. (b) измеренные в геометрии Фарадея для объемного РМП FMR (заштрихованные треугольники) и для образцов РМП CКЯ FM1 (незаштрихованные квадраты) и FM3 (заштрихованные круги). Вставка показывает подуровни основного $F=1$ состояния акцептора $\mathrm{Mn}$, расщепленного в случайных полях и в результате размерного квантования в соответствии с выражением 4.

на одиночном акцепторе $\mathrm{Mn}$ (режим легирования) или с дырками примесной зоны (режим РМП). Заметный сдвиг по энергии $(\Sigma$ в вставке на рис. 1$)$ между возбуждающей энергией и точкой рождения электронов ' 0 ' означает, что как и в объемном ( $\mathrm{Ga}, \mathrm{Mn}) \mathrm{As}[5,6]$ существует заметный энергетический зазор между вершиной валентной зоны и примесной зоной $\mathrm{Mn}$.

Ранее было показано $[5,6]$, что поляризация ГФЛ в магнитном поле определяется спиновой поляризацией дырок, что может быть использовано для изучения $p-d$ обменного взаимодействия и его зависимости от размерного квантования в СКЯ. На рис. 2 показана зависимость циркулярной поляризации ГФЛ от магнитного поля, измеренная в точке рождения электронов '0' для СКЯ, выращенной в режиме легирования (рис. 2, $a$ ) и режиме РМП (рис. 2, b). Чтобы подчеркнуть влияние размерного квантования на поляризацию ГФЛ мы представили зависимости, измеренные на объемном GaAs : Mn (заштрихованные круги на рис. 2,a) и объемном (Ga,Mn)As (4\% Mn) (заштрихованные треугольники на рис. $2, b$ ). Можно видеть, что размерное квантование уменьшает в четыре раза поляризацию ГФЛ в случае легированного образца $D 1$ (или $D 2$ ) (заштрихованные квадраты на рис. 2,a). Наоборот, в объемном (заштрихованные треугольники) и 2D PMП (Ga,Mn)As (незаштрихованные квадраты и заштрихованные круги на рис. $2, b)$ поляризация ГФЛ насыщается приблизительно на одном и том же уровне 0.26. Отметим, что в геометрии Фарадея поляризация насыщения не зависит

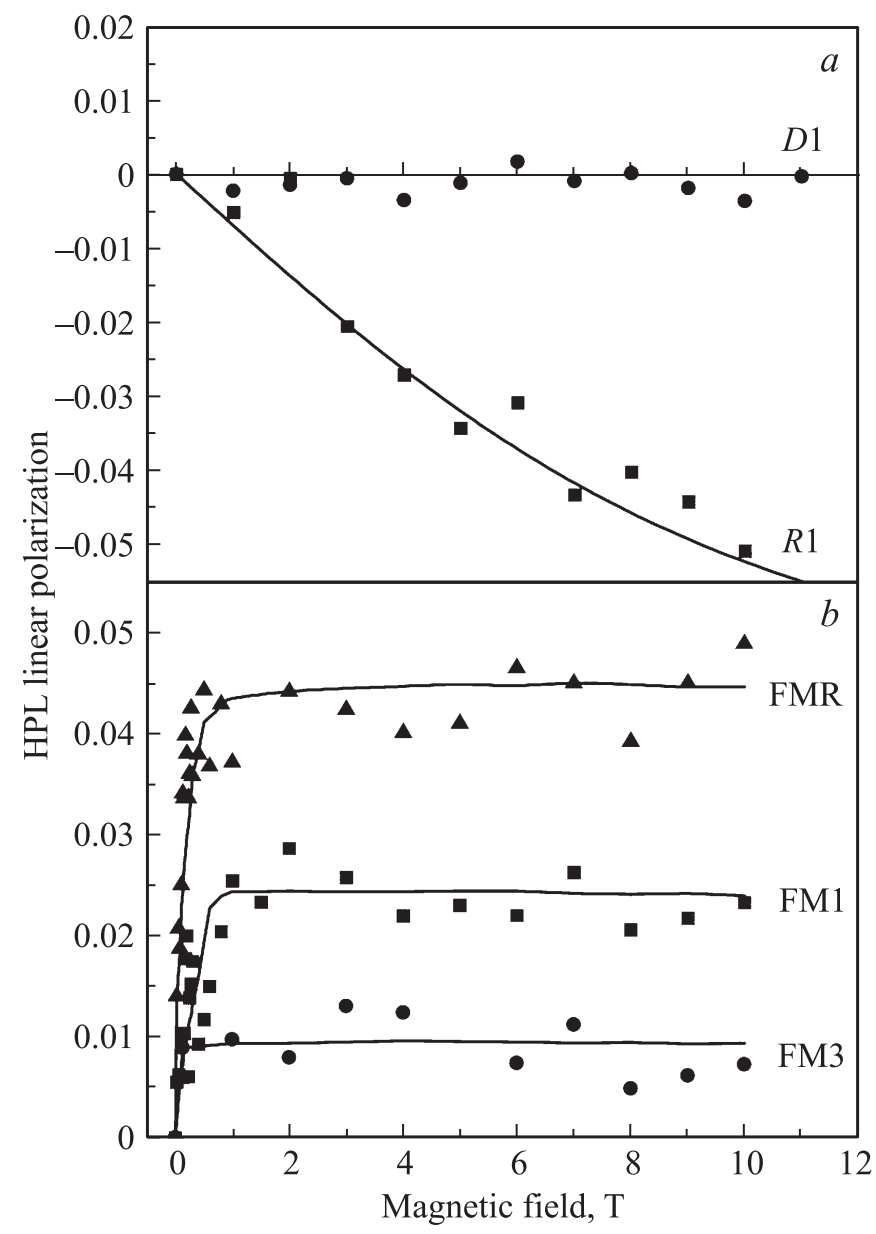

Рис. 3. Зависимости линейной поляризации ГФЛ от магнитного поля: $(a)$ измеренная в геометрии Фойхта при темпеpaтуре $T=5 \mathrm{~K}$ в легированном объемном образце $R 1$ (заштрихованные квадраты) и СКЯ $D 1$ (заштрихованные круги). (b) измеренная в геометрии Фойхта для различных объемных образцов: FMR (заштрихованные треугольники) РМП и СКЯ FM1 (заштрихованные квадраты) и FM3 (заштрихованные круги). 
от ширины КЯ как для РМП (Ga,Mn)As, так и для GaAs : Mn.

Совершенно другое поведение было обнаружено для линейной поляризации ГФЛ при измерении в геометрии Фойхта. На рис. 3, a показана зависимость линейной поляризации от магнитного поля, измеренная на объемном GaAs : Mn (заштрихованные квадраты) и СКЯ GaAs : Mn/AlAs (заштрихованные круги). Аналогичные зависимости для объемного (заштрихованные треугольники) и РМП СКЯ (заштрихованные квадраты и круги) с различными ширинами КЯ показаны на рис. $3, b$. Видно, что в легированном объемном $\mathrm{GaAs}: \mathrm{Mn}$ линейная поляризация ГФЛ насыщается в геометрии Фойхта на уровне $\rho_{l} \sim 0.08$, в то время как в легированной СКЯ степень линейной поляризации близка к 0 (в пределах величины ошибки) во всем диапазоне магнитных полей и не зависит от ширины КЯ. Наоборот, линейная поляризация ГФЛ в ферромагнитном РМП образце FM1 (Ga,Mn)As/AlAs (заштрихованные квадраты на рис. $3, b$ ) вдвое меньше, чем в объемном ферромагнитном РМП и уменьшается с уменьшением ширины КЯ (см. заштрихованные круги на рис. $3, b$ для образца FM3). Такое поведение линейной/циркулярной поляризации ГФЛ, измеренной в геометрии Фарадея/Фойхта в РМП СКЯ, можно объяснить в модели, предполагающей, что дырки связаны на одиночном акцепторе $\mathrm{Mn}$ в легированных КЯ или локализованы в примесной зоне в РМП СКЯ (Ga,Mn)As/AlAs.

\section{4. Обсуждение}

Для начала рассмотрим электронную структуру одиночного акцептора $\mathrm{Mn}$, расположенного в центре КЯ. B KЯ GaAs Mn замещает атом Ga и формирует уровень акцептора с энергией $0.11 \mathrm{eV}$ над потолком валентной зоны. Электронное состояние акцептора определяется $3 d$-оболочкой $\mathrm{Mn}^{-}$, которая в сферическом приближении характеризуется спином $S=5 / 2$ и дыркой, которая в состоянии $1 S_{3 / 2}\left(\Gamma_{8}\right)$ характеризуется эффективным угловым моментом $J=3 / 2$ [8]. Полный угловой момент всей системы $F$ изменяется от $\left|S_{d}-J_{h}\right|=1$ и до $S_{d}+J_{h}=4$ через 1 . Благодаря обменному взаимодействию состояние акцептора расщепляется на четыре подуровня: $F=1,2,3,4$, причем состояние с $F=1$ является основным в случае антиферромагнитного взаимодействия $[8,9]$. Взаимодействие с магнитным полем приводит к расщеплению основного состояния на три подуровня с энергией

$$
E_{F=1}=\mu_{B} g_{F=1} m_{F=1} B,
$$

где $m_{F=1}=0, \pm 1 \quad$ проекция углового момента на магнитное поле, а $g_{F=1}-g$-фактор основного состояния. Волновые функции и $g$-фактор $\left(g_{F=1}=\right.$ $=\left(7 g_{e}-3 g_{h}\right) / 4=11 / 4$, где $g_{h}=-1-g$-фактор дырки, $g_{e}=2-g$-фактор $3 d$ электронов $\mathrm{Mn}$ ) основного состояния были определены в [8]. В этой работе было найдено, что обменное взаимодействие существенно изменяет правила отбора для оптических переходов $e-A_{\mathrm{Mn}}^{0}$.
В частности, только учет обменного взаимодействия в объемном $\mathrm{GaAs}$ уменьшает циркулярную поляризацию в насыщающем магнитном поле $\left(\mu_{B} g_{F=1} B \gg k T\right)$ до величины $5 / 7$ (отметим, что предельное значение поляризации обычного акцептора, без учета обменного взаимодействия, равно $\left.\rho_{c}=1\right)$. Случайные локальные электрические поля или деформации, действующие на акцептор, приводят к дальнейшему уменьшению циркулярной поляризации из-за расщепления основного состояния $F=1[8,9]$.

Можно ожидать, что совместное влияние размерного квантования и случайных полей существенно изменит поляризационные характеристики фотолюминесценции для оптического перехода $e-A_{\mathrm{Mn}}^{0}$.

Действительно, в КЯ основное состояние мелкого акцептора расщеплено на подуровни легких и тяжелых дырок, причем основным является состояние тяжелой дырки. Величина расщепления зависит от ширины КЯ и изменяется в интервале $\Delta \sim 2-7 \mathrm{meV}$ для ширин КЯ в интервале $10-4 \mathrm{~nm}$ [10]. Можно ожидать, что величина расщепления легкая-тяжелая дырка для акцептора $\mathrm{Mn}$ в КЯ 5-10 nm не превысит $\Delta<1-2 \mathrm{meV}$ из-за того, что боровский радиус акцептора $\mathrm{Mn}$ много меньше боровского радиуса мелкого акцептора. Гамильтониан, учитывающий размерное квантование, может быть представлен в виде

$$
H_{\Delta}=\Delta\left(\hat{F}_{z}^{2}-2 / 3\right)
$$

где $F_{z}$ - проекция полного углового момента $(z$ совпадает с направлением роста КЯ). Влияние случайных полей может быть описано гамильтонианом

$$
H_{\delta}=\delta\left(\hat{F}_{X}^{2}-2 / 3\right),
$$

где $\delta$ - энергия расщепления, обусловленная случайными полями, $X-$ направление случайного поля, которое лежит в плоскости $X Y$. Совместное влияние размерного квантования и случайных полей описывается суммой выражений (2) и (3). В отсутствие магнитного поля $B=0$ энергия подуровней может быть представлена в виде (см. вставку на рис. 2)

$$
E_{1}=\frac{-2 \Delta+\delta}{3}, \quad E 2=\frac{\Delta+\delta}{3}, \quad E_{3}=\frac{\Delta-2 \delta}{3} .
$$

В рамках сделанных предположений может быть вычислена линейная и циркулярная поляризация как ГФЛ, так и краевой ФЛ.

В геометрии Фарадея и в случае, когда дырками занят только нижний подуровень '2' (см. вставку на рис. 2, $b$ ) c энергией $E_{2}$, циркулярная поляризация может быть представлена в виде

$$
\rho_{c}^{2}=\beta \frac{5 \epsilon^{2}+2 \epsilon\left(5 \gamma_{1}-4 \gamma_{2}\right)+5 \gamma_{1}^{2}-8 \gamma_{1} \gamma_{2}+5 \gamma_{2}^{2}-9 \gamma_{3}^{2}}{7 \epsilon^{2}+2 \epsilon\left(7 \gamma_{1}-4 \gamma_{2}\right)+7 \gamma_{1}^{2}-8 \gamma_{1} \gamma_{2}+7 \gamma_{2}^{2}+19 \gamma_{3}^{2}}
$$

где $\epsilon=E m_{0} / E_{e} m_{e}$ (здесь $E_{e}-$ кинетическая энергия электронов в зоне проводимости, $E$ - энергия фотона 
ФЛ (см. вставку на рис. 1) и $m_{e}-$ эффективная масса электрона), $\gamma_{1}, \gamma_{2}, \gamma_{3}$ - параметры Латтинжера. Влияние случайных полей в выражении (5) учтено в параметре $\beta=1 / \sqrt{\left(1+\left(\delta / 2 \mu_{B} g_{F=1} B\right)^{2}\right.}$. Излучательная рекомбинация электрона с дыркой, локализованной на подуровне ' 1 ', приводит к рождению неполяризованного фотона $\left(\rho_{c}^{1}=0\right)$, в то время как переход на подуровень '3' сопровождается изменением знака циркулярной поляризации $\left(\rho_{c}^{3}=-\rho_{c}^{2}\right)$. Очевидно, что циркулярная поляризация уменьшается с увеличением случайных полей в плоскости $X Y$ КЯ. Важно отметить, что размерное квантование не влияет на циркулярную поляризацию в геометрии Фарадея, поскольку в этой геометрии проекция углового момента на ось $Z$ (направление роста КЯ) совпадает с направлением магнитного поля. Оценка с параметрами, использованными в эксперименте $\left(E \sim 1.8 \mathrm{eV}, E_{e} \sim 0.3-0.4 \mathrm{eV}\right)$, показывает, что поляризация $\rho_{c}$ заметно уменьшается (от 0.5 до 0.3 ), когда отношение $\delta / 2 \mu_{B} g_{F=1} B$ увеличивается от 1 до 2 . Это уменьшение циркулярной поляризации ГФЛ в СКЯ находится в хорошем согласии с экспериментально наблюдаемым изменением поляризации (рис. 2,a). В этой оценке мы рассматривали только переходы, дающие вклад с наибольшей циркулярной поляризацией (т.е. подуровень ' 2 '). Очевидно, что учет переходов, дающих вклад в неполяризованное излучение (подуровень ' $1{ }^{\prime}$ ) или отрицательно поляризованное (подуровень ' 3 '), только улучшит согласие между теоретической моделью и экспериментом. Отметим, что в случае краевой ФЛ $\left(E_{e}=0\right)$ выражение (5) принимает простую форму

$$
\rho_{c}=5 \beta / 7
$$

В геометрии Фойхта (вставка на рис. 3, $a$ ), в противоположность геометрии Фарадея, размерное квантование в КЯ приводит к уменьшению степени линейной поляризации акцептора $\mathrm{Mn}$. Для нижнего подуровня, занятого дыркой, степень линейной поляризации может быть записана в виде

$$
\begin{aligned}
\rho_{l}=\frac{2 \alpha^{2}\left(3 \gamma_{3}^{2}-\left(\epsilon+\gamma_{1}+\gamma_{2}\right)\left(\epsilon+\gamma_{1}-\gamma_{2}\right)\right)}{2\left(19 \alpha^{2}+11\right) \gamma_{3}^{2}+\left(11 \alpha^{2}+4\right)\left(\epsilon+\gamma_{1}-\gamma_{2}\right)^{2}+} \\
+\left(3 \alpha^{2}+2\right)\left(\epsilon+\gamma_{1}+\gamma_{2}\right)^{2}
\end{aligned}
$$

где $\alpha=\frac{1}{\sqrt{2}}\left[\frac{\Delta}{\mu_{B} g_{F=1} B}-\sqrt{1+\left(\frac{\Delta}{\mu_{B} g_{F=1} B}\right)^{2}}\right]$.

В объемном GaAs или КЯ с пренебрежимо малым эффектом размерного квантования $\alpha=-1 / \sqrt{2}$, линейная поляризация в геометрии Фойхта достигает своего максимального значения $\rho_{l} \approx-0.07$. Расчетные знак и величина поляризации $\rho_{l}$ в объемном случае находятся в хорошем согласии с наблюдаемыми в эксперименте (рис. 3,a). Размерное квантование проявляет себя в значительном уменьшении $\rho_{l}$, как это видно из рис. $3, a$ и $b$ для легированного (D1) и РМП (FM1 и FM3) СКЯ соответственно. Для объяснения этого уменьшения поляри- зации в СКЯ необходимо учесть расщепление основного состояния акцептора Мn из-за размерного квантования (см. (2)). Учет размерного квантования в выражении 7 предсказывает $\rho \sim 0.012$ для $\Delta / \mu_{B} g_{F=1} B=1$. Тенденция линейной поляризации к 0 при уменьшении ширины КЯ явно наблюдается в случае легированных КЯ, а также для РМП СКЯ (см. рис. 3,b). Смена знака линейной поляризации в КЯ по сравнению с объемным случаем происходит из-за обратного порядка подуровней $F=0$ и \pm 1 в СКЯ.

Линейная или циркулярная поляризация, измеренные в геометрии Фойхта или Фарадея (см. рис. 2, $b$ и рис. $3, b)$ в насыщающем магнитном поле, находятся в хорошем согласии с теорией, развитой для одиночного акцептора Mn в СКЯ. Это означает, что в РМП СКЯ дырки локализованы в примесной зоне, которая, как и в объемном случае, формируется в результате перекрытия волновых функций одиночных акцепторов Mn. Отметим, что если бы дырки заполняли валентную зону, следовало бы ожидать значительно большую величину циркулярной поляризации в геометрии Фарадея и нулевую линейную поляризацию в геометрии Фойхта. Такое поведение поляризации связано с тем, что размерное квантование приводит к существенной анизотропии $g$-фактора дырки.

\section{5. Заключение}

Развита модель акцептора Мn в КЯ, описывающая поляризационные характеристики ГФЛ и краевой ФЛ. Анализ спектров ГФЛ и их поляризации в парамагнитных GaAs: Mn/AlAs и ферромагнитных (Ga,Mn)As/AlAs СКЯ показал: размерное квантование существенно модифицирует состояние акцептора Мn. Однако даже в самой узкой КЯ случайные электрические поля или деформация в плоскости КЯ играют важную роль в определении поляризации дырок, связанных на одиночном акцепторе $\mathrm{Mn}$ или примесной зоны. Этот эффект приводит к сильному подавлению циркулярной и линейной поляризации ФЛ, так что максимальная, из-за размерного квантования, величина поляризации ФЛ не может быть достигнута.

Более того, наши экспериментальные данные и теоретический анализ продемонстрировали, что поляризация дырок РMП (Ga,Mn)As/AlAs может быть объяснена только в модели, предполагающей, что дырки локализованы в примесной зоне.

Авторы благодарят C. Herrmann за выращенные образцы, а также G. Paris за ценную техническую помощь.

\section{Список литературы}

[1] H. Ohno, A. Shen, F. Matsukura, A. Oiwa, A. Endo, S. Katsumoto, Y. Iey. Appl. Phys. Lett. 69, 363 (1996).

[2] R.C. Myers, M. Poggio, N.P. Stern, A.C. Gossard, D.D. Awschalom. Phys. Rev. Lett. 95, 017204 (2005). 
[3] M. Poggio, R.C. Myers, N.P. Stern, A.C. Gossard, D.D. Awschalom. Pys. Rev. B 72, 235313 (2005).

[4] M. Tanaka, J. Vac. Sci. Technol. B 16, 2267 (1998).

[5] V.F. Sapega, M. Moreno, M. Ramsteiner, L. Däweritz, K. Ploog. Phys. Lett. 94, 137401 (2005).

[6] V.F. Sapega, M. Ramsteiner, O. Brandt, L. Däweritz, K. Ploog. Phys. Rev. B 73, 137401 (2006).

[7] D. Kolovos-Vellianitis, C. Herrmann, A. Trampert, L. Däweritz, K.H. Ploog. J. Vac. Sci. Technol. B 24, 1734 (2006).

[8] Н.С. Аверкиев, А.А. Гуткин, Е.Б. Осипов, М.А. Рещиков. ФTT 30, 765 (1988).

[9] И.Я. Карлик, И.А. Меркулов, Д.Н. Мирлин, Л.П. Никитин, В.И. Перель, В.Ф. Сапега. ФТТ 24, 3550 (1982).

[10] V.F. Sapega, T. Ruf, M. Cardona, K. Ploog, E.L. Ivchenko, D.N. Mirlin. Phys. Rev. B 50, 2510 (1994). 